\title{
“Controversia Marítima Perú contra Chile”. COMENTARIO A LA SENTENCIA DE LA CORTE INTERNACIONAL DE JUSTICIA DE FECHA 27 DE ENERO DE 2014
}

\section{Elvira Badilla PoBlete*}

\section{1) INTRODUCCIÓN}

La Corte Internacional de Justicia, luego de seis años de juicio ${ }^{1}$ emitió su fallo en este caso incoado por Perú y que fue caratulado por el tribunal "Maritime Dispute (Peru vs. Chile)".

Perú lleva el asunto a la Corte Internacional de Justicia (también la Corte o CIJ) fundado en que ambas partes le han otorgado competencia para conocer del asunto según lo establece el artículo XXXI del Tratado Americano de Soluciones Pacíficas o Pacto de Bogotá suscrito el 30 de abril de 1948. Tanto Chile como Perú son partes en este tratado².

La Corte resolvió que entre el Estado de Perú y Chile, existe una frontera marítima y que esta emana de un acuerdo tácito entre ambos. Esta frontera marítima se extiende hasta una distancia de 80 millas náuticas siguiendo la línea del paralelo de latitud, y desde ese punto hasta las 200 millas la frontera continúa siguiendo una línea equidistante a las costas de ambos Estados.

La Corte igualmente decide que el punto de partida de la frontera marítima única que delimita los espacios marítimos respectivos de Perú y Chile está ubicado en la intersección del paralelo de latitud que pasa por el hito fronterizo No. 1 con la línea de más baja marea.

En este comentario nos referiremos, principalmente, al camino que recorre la Corte para determinar la existencia de un acuerdo tácito sobre

Profesora de Derecho Internacional de la Facultad de Ciencias Jurídicas de la Universidad Católica del Norte, Chile. Licenciada en Derecho y Magíster en Ciencia Jurídica por la Pontificia Universidad Católica de Chile. Correo electrónico: ebadilla@ucn.cl

1 Perú presentó su demanda a la Corte el 16 de enero de 2008.

2 Art. XXXI del Pacto de Bogotá “"De conformidad con el inciso $2^{\circ}$ del artículo 36 del Estatuto de la Corte Internacional de Justicia, las Altas Partes Contratantes declaran que reconocen respecto a cualquier otro Estado Americano como obligatoria ipso facto, sin necesidad de ningún convenio especial mientras esté vigente el presente Tratado, la jurisdicción de la expresada Corte en todas las controversias de orden jurídico que surjan entre ellas y que versen sobre: a) La interpretación de un Tratado; b) Cualquier cuestión de Derecho Internacional; c) La existencia de todo hecho que, si fuere establecido, constituiría la violación de una obligación internacional; d) La naturaleza o extensión de la reparación que ha de hacerse por el quebrantamiento de una obligación internacional" Chile ratificó este tratado el 21 de agosto de 1967. Disponible en: http://www.leychile.cl/Navegar?idNorma=400563\&idVersio $\mathrm{n}=1967-09-06$ Fecha de consulta 18 de abril de 2014. 
frontera marítima y la extensión solo hasta las 80 millas náuticas de esta frontera emanada del acuerdo tácito entre ambos Estados.

\section{2) LAS SOLICITUDES DE LAS PARTES A LA CORTE}

Las peticiones de las partes son evidentemente opuestas ${ }^{3}$, mientras Perú señala que no existe frontera marítima entre ambos Estados y más aún solicita a la Corte que la establezca utilizando el método de la equidistancia de acuerdo al derecho internacional ${ }^{4}$, Chile por su parte indica que existe una frontera marítima entre ambos Estados establecida en la Declaración de Santiago de 1952 y que dicha frontera es la línea del paralelo de latitud.

Tampoco hay coincidencia entre las partes sobre el punto donde se inicia la frontera marítima. Sumado además a que el Estado de Chile niega igualmente los derechos de soberanía reclamados por Perú en el punto (2) indicado infra.

Las peticiones concretas de las partes son las siguientes:

Perú pide a la Corte que resuelva y declare que:

“(1) La delimitación entre las respectivas zonas marítimas entre la República de Perú y la República de Chile es una línea que comienza en el "Punto Concordia" (definido como la intersección con la línea de bajamar de un arco de 10 kilómetros de radio, cuyo centro está en el primer puente sobre el río Lluta del Ferrocarril Arica a La Paz) y equidistante de las líneas de base de ambas Partes, hasta un punto situado a una distancia de 200 millas marinas desde esas mismas líneas de base; y que

3 Corte Internacional de Justicia (CIJ) “Controversia marítima Perú c. Chile” fallo de 27 de enero de 2014. Disponible en www.icj-cij.org Fecha de consulta: 12 de febrero de 2014, p. 15 , párr.22.

4 El principio de la equidistancia para delimitar costas entre Estados adyacentes se encuentra consagrado en diversos tratados: Art.12 .1 de la Convención de Ginebra sobre mar territorial y zona contigua de 29 de abril de 1958: "Cuando las costas de dos Estados se hallen situadas frente a frente o sean adyacentes, ninguno de dichos Estados tendrá derecho, salvo mutuo acuerdo en contrario, a extender su mar territorial más allá de una línea media determinada de forma tal que todos sus puntos sean equidistantes de los puntos más próximos de las líneas de base a partir de las cuales se mide la anchura del mar territorial de cada uno de esos Estados. No obstante, la disposición de este párrafo no será aplicable cuando, por la existencia de derechos históricos o por otras circunstancias especiales, sea necesario delimitar el mar territorial de ambos Estados en otra forma”. Disponible en: https://treaties.un.org/doc/ Publication/UNTS/Volume\%20516/volume-516-I-7477-Other.pdf Fecha de consulta: 2 de marzo de 2014; Artículo 6 de la Convención de Ginebra sobre plataforma continental de 29 de abril de 1958, reitera este principio. Disponible en: https://treaties.un.org/doc/Publication/UNTS/Volume\%20499/volume-499-I-7302-Other.pdf Fecha de consulta: 2 de marzo de 2014; Lo mismo reitera el artículos 15 de la Convención de las Naciones Unidas sobre el Derecho del Mar de 1982. Disponible en http://www.un.org/Depts/los/convention_agreements/texts/unclos/convemar_es.pdf [Fecha de consulta: 19 de febrero de 2014. 
(2) Más allá del punto donde termina el límite marítimo común, el Perú tiene derecho a ejercer derechos de soberanía exclusivos sobre una área marítima hasta una distancia de 200 millas marinas desde sus líneas de base" 5 .

Por su parte Chile, en su oportunidad procesal, solicita a la Corte que

"(a) Deniegue las reclamaciones peruanas en su totalidad;

(b) Resuelva y declare que:

(i) Los derechos de Chile y Perú sobre sus respectivas zonas marítimas han sido íntegramente delimitados por tratados;

(ii) esos derechos sobre las respectivas zonas marítimas están delimitados por una frontera que sigue el paralelo de latitud que pasa por el hito de la frontera terrestre entre Chile y Perú que se encuentra más próximo al mar y que es conocido como Hito No 1, cuya latitud es $18^{\circ} 21^{\prime} 00^{\prime \prime} \mathrm{S}$ referida al Datum WGS84;

(iii) Perú carece de todo derecho sobre zona marítima alguna que se encuentre al Sur de ese paralelo"6.

\section{3) El ACUERdo TÁCito SOBRE DELIMITACIÓN DE LA FRONTERA MARÍTIMA}

La primera tarea que debe cumplir la Corte es determinar si existe una frontera marítima entre ambos Estados como lo ha indicado Chile en respuesta a la petición de Perú.

La Corte analiza y toma postura frente a los acuerdos mencionados por las partes en sus alegaciones así como la práctica también invocada por ambos Estados.

\section{1) Las Proclamaciones de $1947^{7}$}

Sobre estas declaraciones por medio de las que Perú y Chile separadamente reclaman ciertos derechos marítimos, la Corte ha dicho que

5 Vide Memoria presentada por Perú el 20 de marzo de 2009, versión en español disponible en: http://www.rree.gob.pe/noticias/Documents/Memoria\%20del\%20Gobierno\%20 del\%20Per\%C3\%BA.pdf p. 276. Fecha de consulta: 5 de marzo de 2014. La versión original y oficial en inglés está disponible en http://www.icj-cij.org/docket/index.php?p1=3\&p2 $=3 \& \mathrm{k}=88 \&$ case $=137 \&$ code $=$ pch $\& \mathrm{p} 3=1$

6 Vide Contramemoria presentada por el Estado de Chile el 9 de marzo de 2010 versión en Español, disponible en www.minrel.cl p. 262. Fecha de consulta. 20 de febrero de 2014. La versión original y oficial en inglés está disponible en http://www.icj-cij.org/docket/index.ph p?p1 $=3 \& \mathrm{p} 2=3 \& \mathrm{k}=88 \&$ case $=137 \& \operatorname{code}=\mathrm{pch} \& \mathrm{p} 3=1$.

7 En 1947 tanto Chile como Perú de manera unilateral proclamaron ciertos derechos marítimos hasta una extensión de 200 millas marinas desde sus costas. La Declaración de Chile fue hecha por el Presidente de la República Gabriel González Videla el 23 de junio de 1947. Por su parte el Presidente de Perú José Luis Bustamante y Rivero lo hace mediante el Decreto Supremo $\mathrm{N}^{\circ} 781$ de 1 de agosto de 1947, el que lleva la firma del entonces canciller Enrique García Sayán. 
solo coinciden en la importancia de ambas y el contexto histórico en que se producen. Más allá de esto, ambos Estados no coinciden en la interpretación que le dan tanto al contenido de ambas Proclamaciones como al sentido jurídico ${ }^{8}$. Haciendo una revisión de ellas la Corte llega a la conclusión que las partes están de acuerdo en que las Proclamaciones no establecen una frontera marítima internacional, por sí mismas", las partes no expresan su voluntad de fijar los límites laterales de los espacios marítimos reclamados ${ }^{10}$.

\section{2) La DeClaración de Santiago de $1952^{11}$}

Es considerada por ambas partes como un tratado, por lo que la Corte no ocupa mayor tiempo en ello. Sobre lo que sí estima la Corte que debe pronunciarse es si este acuerdo establece o no la frontera marítima entre ambos Estados.

La Corte observa que ambas partes le dan un significado diferente al párrafo IV de la Declaración de Santiago ${ }^{12}$, el que se torna relevante, pues para Chile de dicho párrafo se desprende la frontera marítima entre ambos Estados; la que en este caso es el paralelo de latitud que pasa por el punto en el cual la frontera terrestre entre ellos alcanza el mar. Chile sostiene que el párrafo IV delimita tanto las zonas generales como insulares de los Estados parte, argumentando que la referencia a las islas en esta cláusula es una aplicación específica de una regla generalmente acordada, cuya especificación se explica por la particular importancia de las islas para las circunstancias geográficas de Ecuador. Chile fundamenta esta postura en las Actas de la Conferencia de 1952, donde consta que el delegado de Ecuador pidió que se aclarara que el paralelo, tal como se ha dicho supra era la línea fronteriza y que todos los Estados estuvieron contestes en ello. Chile le otorga a estos hechos el valor de un acuerdo relativo a la conclusión de la Declaración de Santiago de 1952 en el sentido del Art. 31, párr. 2 (a) de la Con-

CIJ, "Controversia Marítima Perú c. Chile" 2014, p. 17, párr. 27.

CIJ, "Controversia Marítima Perú c. Chile" 2014, p. 22, párr. 39.

CIJ, "Controversia Marítima Perú c. Chile” 2014, pp.18-23, párr. 33-44.

En agosto del ańo 1952 los Estados de Chile, Ecuador y Perú se reunieron en la Conferencia sobre la Explotación y Conservación de los Recursos Marinos del Pacífico Sur. En esta conferencia los Estados adoptaron cuatro acuerdos. Uno de ellos es la Declaración de Santiago a que se alude en este apartado. CIJ, "Controversia Marítima Perú c.Chile”2014, p. 14 párr. 20.

12 El citado párrafo dice así "IV) En el caso de territorio insular, la zona de 200 millas marinas se aplicará en todo el contorno de la isla o grupo de islas. Si una isla o grupo de islas pertenecientes a uno de los países declarantes estuviere a menos de 200 millas marinas de la zona marítima general que corresponde a otro de ellos, la zona marítima de esta isla o grupo de islas quedará limitada por el paralelo del punto en que llega al mar la frontera terrestre de los Estados respectivos". Disponible en http://www.difrol.cl/index.php?option=com_conten t\&task=view\&id=34\&Itemid=12 . Fecha de consulta el 2 de mayo de 2014. 
vención de Viena sobre el Derecho de los Tratados de 196913_14. La Corte rechaza este argumento del acuerdo emanado de las Actas de la Conferencia de 1952, solo le otorga valor de trabajos preparatorios a tales actas, que en materia de interpretación de los tratados son un medio complementario para ello en el sentido del artículo 32 de la convención ya mencionada ${ }^{15}$. Sin perjuicio de que el tribunal, finalmente, dice no recurrir a medios complementarios de interpretación, la Corte, tal como hizo en otros fallos, solo les da valor como material relevante ${ }^{16}$.

Desde la perspectiva de Perú, el citado párrafo IV de la Declaración de Santiago de 1952 no establece la frontera marítima lateral entre los Estados ${ }^{17}$. Rechaza también la idea de una delimitación general para ser viable una particular para las islas ${ }^{18}$ y solo le atribuye a las Actas de la Conferencia de Santiago de 1952 el valor de trabajos preparatorios ${ }^{19}$, el mismo valor que finalmente la Corte les atribuyó (ver supra). En conclusión la Corte, respecto de la Declaración de Santiago de 1952 indica que no contiene un acuerdo de frontera marítima lateral en los términos reclamado por Chile ${ }^{20}$. Ahora bien, la Corte en el párrafo inmediatamente anterior al que hemos citado indica la existencia de una "especie de acuerdo" entre Perú y Chile en relación con sus fronteras marítimas. La Corte

13 CIJ, "Controversia Marítima Perú c. Chile” 2014, p. 25, párr.51.

14 El Art. 31 párrafo 2 letra a) de la Convención de Viena sobre Derecho de los Tratados de 1969: "Para los efectos de la interpretación de un tratado, el contexto comprenderá, además del texto, incluidos su preámbulo y anexos: a) todo acuerdo que se refiera al tratado y haya sido concertado entre todas las partes con motivo de la celebración del tratado".

15 "The Court recalls Chile's argument, based on Article 31, paragraph 2 (a), of the Vienna Convention on the Law of Treaties, that the Minutes of the 1952 Conference constitute an "agreement relating to the treaty which was made between all the parties in connection with the conclusion of the treaty". The Court considers that the Minutes of the 1952 Conference summarize the discussions leading to the adoption of the 1952 Santiago Declaration, rather than record an agreement of the negotiating States. Thus, they are more appropriately characterized as travaux préparatoires which constitute supplementary means of interpretation within the meaning of Article 32 of the Vienna Convention on the Law of Treaties" CIJ, "Controversia Marítima Perú c.Chile” 2014, p. 28, párr.65.

16 CIJ, "Controversia Marítima Perú c. Chile" 2014, p. 29, párr. 66.

17 "[...]Peru argues that in so far as the continental coasts of the States parties are concerned, the 1952 Santiago Declaration simply claims a maritime zone extending to a minimum distance of 200 nautical miles, addressing only seaward and not lateral boundaries." CIJ, "Controversia Marítima Perú c. Chile” 2014, p.25, párr. 53.

18 "[...]Peru rejects Chile's argument that a general maritime delimitation must be assumed in paragraph IV so as to make the reference to insular delimitation effective". CIJ, "Controversia Maritima Perú c. Chile” 2014, p.25, párr.54.

19 "[...] Peru contests Chile's interpretation of the Minutes of the 1952 Conference, arguing also that these do not constitute any form of "recorded agreement" but could only amount to travaux préparatoires" CIJ, "Controversia Marítima Perú c. Chile” 2014, p. 26, párr. 54.

20 "The Court has concluded, contrary to Chile's submissions, that Chile and Peru did not, by adopting the 1952 Santiago Declaration, agree to the establishment of a lateral maritime boundary between them along the line of latitude running into the Pacific Ocean from the seaward terminus of their land boundary." CIJ, "Controversia Marítima Perú c.Chile” 2014, p. 30 párr. 70 
menciona que entre otros factores para considerarlo así ha tenido presente, la propuesta Chilena original ${ }^{21}$ y el uso de líneas paralelas como límite de la zona marítima de una isla de un Estado parte localizada a menos de 200 millas náuticas de la zona marítima general de otro Estado parte ${ }^{22}$.

Tras esta conclusión, la Corte analiza los diversos acuerdos de 1954.

\section{3) LOS DIVERSOS ACUERDOS DE 1954}

Bajo este nombre común la Corte se pronuncia sobre tres instrumentos $^{23}$ : La Convención Complementaria a la Declaración de Santiago de 1952; El acuerdo relativo a Medidas de Supervisión y Control de las Zonas Marítimas de los Estados Signatarios y el Convenio relativo a Zona Especial Fronteriza Marítima de 1954. Sobre los dos primeros, la Corte no otorga relevancia a efectos de la tarea principal que se ha propuesto al analizar estos documentos. Es más, respecto al segundo de ellos la Corte expresamente concluye que este no contiene ninguna indicación sobre la naturaleza o la localización de las fronteras de las zonas marítimas de que trata ${ }^{24}$.

La Corte se refiere al análisis más detallado que hace del Convenio sobre Zona Especial Fronteriza Marítima de 195425. Las conclusiones relevantes para la tarea que la Corte emprendió dicen relación con la verificación de que en dicho Convenio los Estados signatarios reconocen que una frontera marítima existía ${ }^{26}$. Si bien no está indicado en este acuerdo internacional cuándo y por qué medios esa frontera fue acordada dice la Corte, el hecho que las Partes reconozcan de manera expresa su existencia la lleva a la conclusión del acuerdo tácito sobre la frontera marítima al que llegaron previamente a la celebración de este Convenio sobre Zona Especial Fronteriza Marítima los Estados. La Corte agrega que ha concluido lo anterior teniendo presente "ciertos elementos" de las Proclamaciones de 1947 y la Declaración de Santiago de $1952^{27}$ i.e., para las Proclamaciones

21 CIJ, “Controversia Marítima Perú c. Chile” 2014, p.29, párr. 67.

22 La Corte dice: "[...] suggest that there might have been some sort of shared understanding among the States parties of a more general nature concerning their maritime boundaries." CIJ, "Controversia Marítima Perú c. Chile” 2014, p 30, párr. 69. (la negrilla es nuestra). "[...]Of these six Agreements only the 1954 Complementary Convention and the 1954 Special Maritime Frontier Zone Agreement were given any real attention by the Parties in the course of these proceedings, except for brief references by Chile to the Supervision and Control Convention [...]”. CIJ. "Controversia Marítima Perú c. Chile” 2014, p. 31, párr. 73.

24 CIJ, "Controversia Marítima Perú c. Chile” 2014, p. 33 párr. 79.

25 CIJ, "Controversia Marítima Perú c. Chile” 2014, pp. 33-37 párr. 80-92.

26 CIJ, "Controversia Marítima Perú c. Chile” 2014, p. 36 párr. 90.

$27 \quad$ La Corte dice en el párrafo 91 de su fallo: "The 1954 Special Maritime Frontier Zone Agreement does not indicate when and by what means that boundary was agreed upon. The Parties' express acknowledgment of its existence can only reflect a tacit agreement which they had reached earlier. In this connection, the Court has already mentioned that certain elements of the 1947 Proclamations and the 1952 Santiago Declaration suggested an evolving understanding between the Parties concerning their maritime boundary [...] La Corte concluye enfática, luego de 
la necesidad de establecer una frontera marítima lateral a futuro ${ }^{28}$ y para la Declaración de 1952 la existencia de una "especie de acuerdo" de una naturaleza más general sobre sus fronteras marítimas a partir del uso de líneas paralelas como límite de la zona marítima de las islas ${ }^{29}$.

A similar conclusión llega el tribunal a partir de los arreglos sobre la situación y construcción de los faros de 1968-1969 entre Perú y Chile. La Corte si bien considera que los arreglos para la instalación de los faros nada dice sobre la frontera marítima, sí estima que es posible verificar que los arreglos se llevaron adelante basados en que ya existía una frontera marítima que se extendía a lo largo del paralelo, más allá de 12 millas náuticas ${ }^{30}$.

De esta manera la Corte luego de la revisión de las normas convencionales que el Estado de Chile invoca como prueba de la existencia de la frontera marítima entre los dos Estados, concluye que estos instrumentos citados dan cuenta de un acuerdo tácito de frontera marítima, el que de manera expresa se refleja en el Convenio sobre Zona Especial Fronteriza Marítima de 1954.

Mal podría haber resuelto la Corte una cuestión diferente en este punto, por cuanto no cabe cuestionarse la existencia de una frontera marítima frente a la existencia de un acuerdo que pretende evitar violaciones a una frontera marítima, transgresión que solo puede ocurrir en la medida que exista aquella. El Convenio de 1954, lo dice en su preámbulo "[...] violaciones de la frontera marítima entre los Estados vecinos [...]" y posteriormente en el artículo primero y esencial del acuerdo no hace más que añadir claridad al asunto: "Establécese una Zona Especial, a partir de las 12 millas marinas de la costa, de 10 millas marinas de ancho a cada lado del paralelo que constituye el límite marítimo entre los dos países"31. Lo mismo podemos expresar para el arreglo de los faros mencionados supra, por cuanto en el documento firmado por ambos Estados declaraban como propósito la instalación de señales visibles desde el mar para materializar el paralelo de la frontera marítima ${ }^{32}$.

No es primera vez que la Corte ha formulado la idea del acuerdo tácito entre los Estados relacionado a la soberanía territorial. En el caso con-

citar su propia jurisprudencia sobre acuerdos tácitos en materia de fronteras: "In this case, the Court has before it an Agreement which makes clear that the maritime boundary along a parallel already existed between the Parties. The 1954 Agreement is decisive in this respect. That Agreement cements the tacit agreement". (la negrilla es nuestra). CIJ, "Controversia Marítima Perú c. Chile" 2014, pp. 36-37, párr. 91

28 CIJ, "Controversia Marítima Perú c. Chile" 2014, p.22, párr. 43.

29 CIJ, "Controversia Marítima Perú c. Chile" 2014, p.30 párr. 69.

30 CIJ, "Controversia Marítima Perú c. Chile” 2014, p.39, párr. 99

31 Vide Convenio sobre Zona Especial Fronteriza Marítima de 4 de diciembre de 1954 Disponible en : http://www.difrol.cl/index.php?option=com_content\&task=view\&id=35\&Itemid=12

32 CIJ, "Controversia Marítima Perú c.Chile" 2014, p. 38, párr.96. 
cerniente a soberanía territorial entre Malasia y Singapur (2008) la Corte ha dicho que cualquier transferencia de soberanía puede ser hecha por un acuerdo entre los Estados involucrados. Atendido que el derecho internacional no impone formas a estos acuerdos, la Corte no reduce la expresión acuerdo a los tratados formales ${ }^{33}$, aceptando que este puede emanar de la conducta de los Estados, así lo ha expresado en su fallo: "Any passing of sovereignty might be by way of agreement between the two States in question. Such an agreement might take the form of a treaty [... T The agreement might instead be tacit and arise from the conduct of the Parties. International law does not, in this matter, impose any particular form" 34 .

En la controversia territorial y marítima entre Nicaragua y Honduras (2007) la Corte se pronuncia sobre la existencia de un acuerdo tácito entre las partes sobre la materia en controversia y establece un estricto estándar de prueba (que no es alcanzado): "The Court must now determine whether there was a tacit agreement sufficient to establish a boundary. Evidence of a tacit legal agreement must be compelling. The establishment of a permanent maritime boundary is a matter of grave importance and agreement is not easily to be presumed"35. La Corte se ha referido recientemente en el caso Nicaragua contra Colombia (2012) ${ }^{36}$ sobre la idea de un acuerdo tácito reiterando lo dicho en su fallo entre Nicaragua y Honduras (2007).

El Tribunal Internacional del Derecho del Mar (ITLOS, por sus siglas en inglés) en los párrafos 100 a 118 de su fallo de marzo de 2012, en el caso sobre delimitación de la frontera marítima entre Bangladesh y Myanmar en la Bahía de Bengala, refiriéndose a un acuerdo tácito entre los Estados en conflicto, se remite a los dichos de la Corte Internacional de Justicia sobre la materia ${ }^{37}$. Sigue la jurisprudencia de la Corte Internacional de Justicia que si bien un acuerdo tácito sobre delimitación de una

33 De acuerdo al art. 2 de la Convención de Viena sobre Derecho de los Tratados de 1969, "se entiende por tratado un acuerdo internacional celebrado por escrito entre Estados y regido por el derecho internacional ya conste en un instrumento único o en dos o más instrumentos conexos y cualquiera que sea su denominación particular”.

34 Sovereignty over Pedra BrancalPulau Batu Puteh, Middle Rocks and South Ledge (Malaysial Singapore),Judgment, I.C.J. Reports 2008, p. 12 Disponible en: file:///E:/A\%20BIBLIOGRAFIA/CASOS\%20CIJ\%20y\%20CPJI/Soberania\%20sobre\%20PedraBranca\%20MALASIA\%20CON\%20SINGAPUR\%202008.pdf Fecha de consulta: 20 de abril de 2014.

35 Territorial and Maritime Dispute between Nicaragua and Honduras in the Caribbean Sea (Nicaragua v. Honduras),Judgment, I.C.J. Reports 2007, p. 753, párr.. 253. Disponible en: http://www.icj-cij.org/docket/files/120/14075.pdf Fecha de consulta: 2 de mayo de 2014.

36 Territorial and Maritime Dispute (Nicaragua v. Colombia),Judgment, I.C.J. Reports 2012, p. 705, párr 219. Disponible en: http://www.icj-cij.org/docket/files/124/17164.pdf Fecha de consulta: 22 de abril de 2014 .

37 Dispute concerning delimitation of the maritime boundary between Bangladesh and Myanmar in the Bay of Bengal fallo de 14 de marzo de 2012, Tribunal Internacional del Derecho del Mar pp. 39-43, párrs. 110-118. Disponible en: https://www.itlos.org/fileadmin/itlos/documents/cases/case_no_16/C16_Judgment_14_03_2012_rev.pdf Fecha de consulta: 22 de abril de 2014 . 
frontera marítima (o terrestre) debe estar fundado en pruebas convincentes, pues estamos frente a un asunto de mucha gravedad, es factible que ello ocurra. Así ha quedado demostrado en el fallo que comentamos.

¿Es este un precedente importante en la jurisprudencia de la Corte? Sí que lo es. Al parecer la Corte estimó que para este caso particular si se cumple con el riguroso estándar de prueba de un acuerdo tácito que ella exigió en casos anteriores como mencionamos supra.

Entre las opiniones disidentes; declaraciones individuales y opiniones separadas que se acompańaron a la sentencia de la Corte por 11 de los 16 magistrados que concurrieron al fallo, ninguno de ellos se pronuncia sobre la imposibilidad de establecer una frontera marítima fundada en un acuerdo tácito. Solo hay quienes rechazan, que para este caso en particular, se haya logrado demostrar un acuerdo tácito ${ }^{38}$.

El juez Bernardo Sepúlveda-Amor sostiene que no hay prueba convincente para establecer la existencia de un acuerdo tácito de frontera marítima entre Perú y Chile, sin embargo expresamente reconoce que no es contrario a la existencia de una frontera marítima fundada en un acuerdo tácito ${ }^{39}$. El magistrado Hisashi Owada ${ }^{40}$ en relación con esta parte del fallo indica su total desacuerdo y su preocupación por cuanto no se

38 Los jueces Xue, Gaja, Bhandari y el juez ad-hoc Orrego Vicuña en su opinión disidente conjunta al fallo de 27 de febrero de la Corte, consideran que el párrafo IV la Declaración de Santiago de 1952 expresa cual es la frontera marítima lateral de los Estados, lo que sumado a otros antecedentes les hacen concluir que la extensión de esta frontera consagrada en esta Declaración es hasta las 200 millas naúticas: "The text of paragraph IV of the 1952 Santiago Declaration implies that the parallel that passes through the point where the land frontier reaches the sea represents the lateral boundary of the general maritime zones of the Parties, which, on the basis of the Parties' maritime claims as pronounced in the Santiago Declaration, extends for 200 nautical miles. Some subsequent agreements concluded between the Parties confirm this interpretation of the Declaration, in particular the 1954 Agreement, the 1955 Protocol and the 1968 agreement. These instruments provide a solid legal basis for the existence of a maritime boundary that extends along the parallel for 200 nautical miles from the continental coasts of Peru and Chile" Disponible en: http://www.icj-cij.org/docket/files/137/17946.pdf p.7 Fecha de consulta: 5 de mayo de 2014.

39 Sepúlveda-Amor, Bernardo (2014) Declaración al fallo de 27 de enero de 2014 de la Corte Internacional de Justicia "Controversia marítima (Perú c. Chile)" p. 1 "As a matter of principle, I do not take issue with the proposition that, in appropriate circumstances, a maritime boundary may be grounded upon tacit agreement" Disponible en http://www.icj-cij.org/docket/files/137/17940.pdf Fecha de consulta: 25 de abril de 2014.

40 Owada, Hisashi (2014) Opinión separada al fallo de 27 de enero de 2014 de la Corte Internacional de Justicia "Controversia marítima (Perú c. Chile)" p. 4 "In my view, this language, in its plain meaning, does not, as such and without additional evidence, warrant the existence of a tacit agreement establishing such a boundary for all purposes between the Parties. Tacit agreements establishing any type of international boundary, either land or maritime, are exceptional for the simple reason that when it comes to the question of territorial sovereignty, States almost always are extremely jealous of safeguarding their sovereignty, and, in a situation involving the issue of transfer of territorial sovereignty, normally act with particular care and caution. It is for this reason that the Court has always adopted a sceptical view towards the claim by a State that a tacit agreement exists establishing a maritime boundary in its favor" Diponible en http:// www.icj-cij.org/docket/files/137/17942.pdf Fecha de consulta 25 de abril de 2014. 
cumpliría con el estándar de prueba exigido por la Corte en el caso entre Nicaragua con Honduras (2007) ya citado. La misma postura adopta la jueza Julia Sebutinde en su opinión disidente ${ }^{41}$.

La Sentencia de la Corte resuelve el asunto por una vía inesperada para ambas partes. Es indudable que la declaración individual que se adjunta al fallo por la jueza norteamericana Joan E. Donoghue, quien afirma que este es un ejemplo de aquellos casos en que ninguna de las partes ha convencido al tribunal con sus alegaciones ${ }^{42}$ algo de ello nos evidencia. Ninguna de las partes se puso en el supuesto del acuerdo tácito. Estimamos que este hecho influyó decisivamente, tal como expresa la jueza Donoghue, en la prueba de la extensión de dicha frontera como veremos en párrafos posteriores: "because the Parties did not address the existence or terms of such an agreement, they did not present evidence focused specifically on the extent of a tacitly-agreed maritime boundary" 43 .

\section{4) LA FRONTERA MARÍTIMA DE 80 MILLAS NÁUTICAS}

Para efectos de determinar la extensión de la frontera marítima convenida de manera tácita y que se encuentra explicitada en el Convenio de 1954 -el que nada dice al respecto- la Corte revisa la práctica de los Estados teniendo en consideración que el citado convenio refleja un propósito particular, por tanto, dice la Corte que la frontera marítima cuya existencia reconoce a lo largo del paralelo, no podría extenderse más allá de aquel espacio en que las partes a la época pudieron realizar actividades de pesca ${ }^{44}$. Bajo esta premisa la Corte estimó que la frontera marítima que sigue la línea del paralelo, solo es posible extenderla hasta las $80 \mathrm{mi}$ llas náuticas.

Para llegar a tal conclusión el tribunal examina una serie de antecedentes que permiten configurar la práctica de ambas partes en litigio, la práctica de terceros Estados y el desarrollo del Derecho internacional de la época.

A partir de los datos aportados por Chile ${ }^{45}$ sobre las actividades de pesca realizada por ambos Estados a inicios de la década de 1950 la Cor-

41 Sebutinde, Julia (2014) Opinión disidente al fallo de 27 de enero de 2014 de la Corte Internacional de Justicia "Controversia marítima (Perú c. Chile”) p. 2 Disponible en: http:// www.icj-cij.org/docket/files/137/17952.pdf Fecha de consulta 25 de abril de 2014.

42 Donoghue, Joan E. (2014) Declaración al fallo de fecha 27 de enero de 2014 de la Corte Internacional de Justicia sobre "Controversia Marítima Perú c. Chile” Disponible en: http:// www.icj-cij.org/docket/files/137/17948.pdf Fecha de Consulta 12 de abril de 2014.

43 Donoghue, Joan E. (2014) p.1.

44 CIJ, "Controversia Marítima Perú c. Chile” 2014, p. 39, párr. 103.

45 Chile acompaña estadísticas confeccionadas por la Organización de las Naciones Unidas para la Alimentación y la Agricultura (FAO). Si bien las estadísticas de la FAO aportadas por Chile menciona la caza de Ballena, no da información del lugar en que se realizó. Vide n. 44. 
te extrae dos conclusiones relevantes a nuestro juicio, el primero es que la actividad pesquera de ambos Estados en esos ańos, era limitada y el segundo, que dicha actividad era principalmente volcada a dos especies (bonito y atún $)^{46}$, las que según declaraciones oficiales de Perú, se encontraban entre las millas 20 a $80^{47}$ de las costas.

La práctica particular de ambos Estados no es contradictoria con la revisada por la Corte en un contexto más general, el reconocimiento por parte de la comunidad internacional de las 200 millas marinas de zona económica exclusiva es un hecho que se consolida con la Convención de Naciones Unidas sobre el Derecho del Mar de 1982. La Corte es exacta en esto.

En los comentarios realizados por los gobiernos en el año 1957 al proyecto de artículos relativos al Derecho del Mar, adoptado por la Comisión de Derecho Internacional de las Naciones Unidas en su octavo período de sesiones, queda en evidencia la discusión de los Estados en torno a este espacio marítimo. Chile declara su preocupación por dicho espacio el que a la época se entendía como alta mar, más allá del mar territorial. Claramente no estaba aún consagrado ni reconocido por la comunidad internacional. Chile indicó en su comentario: "It is not sufficient to recognize the special interest of the coastal State in the maintenance of the productivity of the living resources in any area of the high seas adjacent to its territorial sea it is also necessary to proclaim the coastal State's right to conserve the marine resources in a zone lying off its coasts which is delimited in the light of technical or scientific considerations" 48 .

También es considerado como elemento que apoya la tesis de las 80 millas sostenida por la Corte, la actividad que llevaron a cabo los Estados en cuanto a la detención o incidentes de embarcaciones que violaban la frontera marítima.

En relación a las embarcaciones de Perú y Chile, no hay registros de detenciones de naves que provean las partes o incidentes de naves que se encontraran más allá de las 60 millas náuticas de la costa en el período que le interesa al tribunal, esto es, principalmente a inicios de 1950. Es más, la práctica proporcionada a la Corte hasta 1980 revela que los inci-

46 CIJ, “Controversia Marítima Perú c. Chile” 2014, p. 41, párr. 107.

47 CIJ, "Controversia Marítima Perú c. Chile” 2014, pp. 40-41, párr. 106

48 Carta de la Misión Permanente de Chile ante las Naciones Unidas de 19 de julio de 1957, recogida en Comments by Governments on the Draft Articles Concerning the Law of the Sea Adopted by the International Law Commission at Its Eighth Session. Extract from the Offcial Records of the United Nations Conference on the Law of the Sea, Volume I (Preparatory Documents). La misma idea subyace en los comentarios realizados por el gobierno de Perú mediante carta del Ministro de Relaciones Exteriores de Perú de 5 de agosto de 1957 pp. 97-98. Revisados los comentarios es meridiano que no existía la idea de una zona económica exclusiva de 200 millas marinas como la entendemos hoy, luego de la Convención de Naciones Unidas sobre el Derecho del Mar de 1982. El documento citado está disponible en: http://legal.un.org/diplomaticconferences/lawofthesea-1958/docs/english/vol_I/8_ACONF-13-5_PrepDocs_vol_I_e.pdf Fecha de consulta 29 de marzo de 2014. 
dentes entre ambos Estados ocurrieron a distancias mucho más cercanas a las Costas $^{49}$.

Sobre arrestos o incidentes ocurridos entre la partes después de 1984 y hasta 1994 no ocurrieron más allá de las 40 millas náuticas. Solo a partir de 1996 los arrestos fueron hechos frecuentemente más allá de la milla 60. La Corte destaca que para efectos de la postura chilena, Perú no protestó frente a estos arrestos ${ }^{50}$.

Sobre los incidentes que involucraban naves de terceros Estados, la prueba proporcionada tampoco colabora a la idea de una práctica de los Estados en estas cuestiones, que se extendiera más allá de las 80 millas náuticas. Para estos efectos, Chile presenta un informe de la Secretaría General de la Comisión Permanente del Pacífico Sur que abarca 20 años (1951-1971). Para los primeros 10 años, según el reporte presentado, dice la Corte, está incompleto. El único dato claro que se puede colegir de la información que la Corte declara tener es que, salvo la flota Onassis, que fue detenida en la milla 126 (en la década de 1950, ver supra) el resto de las situaciones no habrían ocurrido más allá de la milla 60 de la costa de Perú ${ }^{51}$.

La Corte menciona que al ser interrogados por un Miembro de la Corte, ambos Estados, reconocieron que su reclamación formulada en la Declaración de Santiago de 1952 (sobre las 200 millas náuticas) no correspondía al derecho internacional de la época y que, inicialmente al menos, no era oponible a terceros Estados ${ }^{52}$.

Lo anterior es coincidente con la observación que hace la Corte tras verificar que la frontera marítima acordada de manera tácita solo se prolonga hasta la milla 80 , en cuanto a que en las aguas más alejadas de sus costas, la preocupación de los Estados que suscribieron el Convenio de 1954 sobre Zona Especial Fronteriza Marítima y que también se reflejó en los instrumentos de 1947 y de 1952, sus actuaciones en 1954 y 1955 e igualmente en el proceso que llevó a las convenciones de Naciones Unidas sobre el derecho del Mar de 1958, era la de tener una posición solidaria, especialmente frente aquellos terceros Estados que practicaban la pesca en alta $\operatorname{mar}^{53}$. Estimamos que similar preocupación se desprende de los comentarios emitidos por ambos Estados en relación con el proyecto de artículos de la Comisión de Derecho Internacional mencionado supra (Vide n. 48).

De la lectura del fallo es posible entender que la Corte no extiende la frontera hasta la milla 200 pues la prueba proporcionada por las partes y considerada relevante por la Corte permite extender dicha frontera -con

\footnotetext{
49 CIJ, “Controversia Marítima Perú c. Chile” 2014, p. 48, párr. 128.

50 CIJ, "Controversia Marítima Perú c. Chile” 2014, p. 53, párrs. 146-147

51 CIJ, "Controversia Marítima Perú c. Chile” 2014, p. 47, párr. 127.

52 CIJ, "Controversia Marítima Perú c. Chile” 2014, p. 44, párr. 116.

53 CIJ, "Controversia Marítima Perú c. Chile” 2014, p.54. párr. 150.
} 
dificultades estimamos- no más allá de la cuestionada milla $80^{54}$. Las partes no pudieron probar la extensión de una frontera tácita. Nos remitimos nuevamente aquí a los dichos de la jueza Donoghue reproducidos en el apartado 3.

\section{5) OBSERVACIONES ADICIONALES}

\section{1) EL PUNTO CONCORDIA}

La Corte si bien se inclina por la postura chilena sobre el inicio de la frontera marítima, indicando que esta tiene como punto de partida la intersección del paralelo de latitud que pasa por el hito $\mathrm{N}^{\circ} 1$ con la línea de bajamar, no se pronuncia por la ubicación del punto "Concordia" donde comienza la frontera terrestre, al que las partes hacen referencia en sus alegaciones, pues no le compete ha dicho, ya que las partes no han formulado esto en sus peticiones 55 .

El proceso ha puesto en evidencia que las partes no coinciden en la ubicación del punto exacto donde se inicia la frontera terrestre entre ellas. Esta frontera está establecida por el tratado de Lima de $1929^{56}$. A su vez el juez ad hoc Gillaume, designado por Perú según ya se expuso, se ha sumado a la decisión mayoritaria de la Corte sobre el punto de inicio de la frontera marítima, pero destaca en su Declaración individual al fallo que esto "[...] in no way prejudges "the location of the starting-point of the land boundary identified as 'Concordia' in Article 2 of the 1929 Treaty of Lima"57.

\section{2) LA EXPRESIÓN “DOMINIO MARÍTIMO”}

En la discusión sobre la determinación de la frontera marítima más allá de la milla 80 , es decir en aquel tramo donde la Corte utilizó la línea

54 Luego de la lectura del fallo, varios medios de prensa tanto nacionales como extranjeros recogieron opiniones de especialistas al respecto, inter alia, Ximena Fuentes, asesora jurídica de la defensa chilena, en Revista Capital online 21 de febrero de 2014, disponible en: http:// www.capital.cl/capital-legal/el-fallo-de-la-haya-abogado-de-su-propia-causa/ ; Alfredo Moreno, como Canciller y Alberto van Klaveren como agente chileno ante la Corte Internacional de Justicia en La Tercera.com 28 de enero de 2014, disponible en: http://www.latercera.com/ noticia/politica/2014/01/674-562941-9-moreno-y-van-klaveren-reafirman-que-decision-dela-haya-sobre-las-80-millas.shtml.

55 CIJ, "Controversia Marítima Perú c. Chile" 2014, p. 58, párr. 163 "The Court observes that a considerable number of the arguments presented by the Parties concern an issue which is clearly not before it, namely, the location of the starting-point of the land boundary identified as "Concordia" in Article 2 of the 1929 Treaty of Lima".

56 Las posiciones divergentes de las partes sobre este punto pueden ser revisadas en los párrafos 155 a 157 del fallo que comentamos.

57 Guillaume, Gilbert (2014) Declaración al fallo de 27 de enero de la Corte Internacional de Justicia "Controversia Marítima (Perú c. Chile)" p. 3 párr. 15. 
equidistante a las costas de ambos Estados hasta las 200 millas náuticas, se evidencia la utilización de la expresión "dominio marítimo en el derecho nacional por parte de Perú.

El Agente peruano, declaró formalmente en representación de su gobierno que dicha expresión usada en su Constitución Política debe entenderse que es aplicada de manera consistente con las zonas marítimas que consagra la Convención de Naciones Unidas sobre el Derecho del Mar de 1982.

La Corte toma nota de esta declaración diciendo que expresa un compromiso formal por parte de Perú ${ }^{58}$.

Estimamos que esta declaración está revestida de gran importancia: Perú no es parte de la Convención de 1982; esta declaración debe tenerse presente al momento de dar cumplimiento al fallo por parte de Perú. 